\title{
Research Article \\ Fixed Points of Multivalued G-Nonexpansive Mappings in Hadamard Spaces Endowed with Graphs
}

\author{
Bancha Panyanak (iD \\ Data Science Research Center, Department of Mathematics, Faculty of Science, Chiang Mai University, Chiang Mai 50200, Thailand
}

Correspondence should be addressed to Bancha Panyanak; bancha.p@cmu.ac.th

Received 27 February 2020; Accepted 20 April 2020; Published 15 May 2020

Guest Editor: Antonio Francisco Roldan Lopez de Hierro

Copyright (C) 2020 Bancha Panyanak. This is an open access article distributed under the Creative Commons Attribution License, which permits unrestricted use, distribution, and reproduction in any medium, provided the original work is properly cited.

\begin{abstract}
A fixed-point theorem for multivalued $G$-nonexpansive mappings in Hadamard spaces is proved. The strong convergence theorems of Browder's and Halpern's iterations are also established. Our results generalize and improve the results of Tiammee et al. (2015), Alfuraidan and Khamsi (2015), Anakkamatee and Tongnoi (2019), and many others.
\end{abstract}

\section{Introduction}

Fixed-point theory is an important tool for finding solutions of problems in the form of equations or inequalities. One of the fundamental and celebrated results in metric fixedpoint theory is the so-called Banach contraction principle which states that every contraction mapping on a complete metric space always has a unique fixed point (see [1]).

In 1967, Browder [2] applied the Banach contraction principle to prove the strong convergence of an implicit iteration for nonexpansive mappings in Hilbert spaces. Later on, Halpern [3] used Browder's result to prove the strong convergence of an explicit iteration for nonexpansive mappings in Hilbert spaces. Notice that both Browder's and Halpern's iterations can be applied to convex optimization problems, variational inequality problems, and image recovery problems (see, e.g., [4]).

In 2008, by combining the concepts of fixed-point theory and graph theory, Jachymski [5] defined a single-valued $G$ contraction mapping on a metric space and proved a generalization of the Banach contraction principle in a complete metric space endowed with a graph. In 2015, Tiammee et al. [6] gave the analogous results of Browder and Halpern in Hilbert spaces endowed with graphs. Recently, Anakkamatee and Tongnoi [7] extended Tiammee et al.'s results from Hilbert spaces to the general setting of Hadamard spaces.

Fixed-point theory for multivalued mappings has many useful applications in applied sciences, for instance, in game theory and optimization theory. It is natural to study the extensions of the known fixed-point results for singlevalued mappings to the setting of multivalued mappings. In 2010, Beg et al. [8] defined a multivalued G-contraction mapping on a metric space and proved the existence of fixed points for such kind of mappings in metric spaces endowed with graphs. In 2015, Alfuraidan and Khamsi [9] introduced the concept of multivalued $G$-nonexpansive mappings as well as the concept of $G$-compact sets and obtained a fixed-point theorem for a multivalued $G$-nonexpansive mapping defined on a $G$-compact convex subset of a complete hyperbolic metric space endowed with a graph.

In this paper, by using the concept of property $G$ introduced by Tiammee et al. [6], we can prove the existence of fixed points for multivalued $G$-nonexpansive mappings in Hadamard spaces endowed with graphs. We also show that, under some appropriate conditions, the sequence of Halpern's iteration converges strongly to a fixed point of a certain multivalued $G$-nonexpansive mapping. Our results generalize and improve many results in the literature.

\section{Preliminaries}

Let $G$ be a directed graph with a set of vertices $V(G)$ and a set of edges $E(G)$ which contain all loops; that is, $(x, x) \in E(G)$ for every $x \in V(G)$. We also assume that $G$ has no parallel edges. Let $x, y \in V(G)$. We say that $x$ dominates $y$ if $(x, y) \in$ 
$E(G)$. Let $A$ and $B$ be nonempty subsets of $V(G)$. We say that $A$ dominates $B$ if $(a, b) \in E(G)$ for all $a \in A$ and $b \in B$.

Let $(M, d)$ be a metric space and $D$ be a nonempty subset of $M$. We denote by $\mathrm{CB}(D)$ the family of nonempty closed bounded subsets of $D$ and by $K(D)$ the family of nonempty compact subsets of $D$. Let $H(\cdot, \cdot)$ be the Hausdorff distance on $\mathrm{CB}(D)$, i.e.,

$H(A, B)=\max \left\{\sup _{a \in A} \operatorname{dist}(a, B), \sup _{b \in B} \operatorname{dist}(b, A)\right\}, A, B \in \mathrm{CB}(D)$,

where dist $(a, B):=\inf \{d(a, b): b \in B\}$ is the distance from the point $a$ to the set $B$.

A multivalued mapping $S: D \longrightarrow C B(D)$ is called a contraction if there exists $k \in[0,1)$ such that

$$
H(S(x), S(y)) \leq k d(x, y) \text { for all } x, y \in D
$$

Moreover, if (2) is valid when $k=1$, then $S$ is called nonexpansive. It is clear that every contraction mapping is nonexpansive and, in general, the converse is not true.

Let $G=(V(G), E(G))$ be a directed graph. Throughout this paper, we assume that $V(G)$ is a nonempty subset of $D$. A multivalued mapping $S: D \longrightarrow C B(D)$ is said to be monotone increasing $G$-contraction [9] if there exists $\lambda \epsilon$ $[0,1)$ such that for any $x, y \in D$ with $(x, y) \in E(G)$ and any $x^{\prime} \in S(x)$, there exists $y^{\prime} \in S(y)$ such that

$$
\begin{gathered}
\left(x^{\prime}, y^{\prime}\right) \in E(G), \\
d\left(x^{\prime}, y^{\prime}\right) \leq \lambda d(x, y) .
\end{gathered}
$$

If (3) is valid when $\lambda=1$, then $S$ is called monotone increasing $G$-nonexpansive. Obviously, every monotone increasing G-contraction mapping is monotone increasing $G$-nonexpansive.

Proposition 1. Let $G=(V(G), E(G))$ be a directed graph such that $V(G)=D$ and $E(G)=D \times D$. Let $S: D \longrightarrow K(D)$ be a multivalued mapping. Then, the following statements hold:

(i) $S$ is contraction if and only if $S$ is monotone increasing G-contraction

(ii) $S$ is nonexpansive if and only if $S$ is monotone increasing G-nonexpansive

Proof. This proof is patterned after the proof of Proposition 1 in [10].

(i) Suppose that $S$ is a contraction mapping with a constant $k \in[0,1)$. Let $x, y \in D$ with $(x, y) \in E(G)$ and let $x^{\prime} \in S(x)$. Since $S(y)$ is compact, there exists $y^{\prime} \in S(y)$ such that

$$
d\left(x^{\prime}, y^{\prime}\right)=\operatorname{dist}\left(x^{\prime}, S(y)\right) \leq H(S(x), S(y)) \leq k d(x, y)
$$

Since $E(G)=D \times D,\left(x^{\prime}, y^{\prime}\right) \in E(G)$. This shows that $S$ is monotone increasing $G$-contraction. Conversely, suppose that $S$ is a monotone increasing $G$-contraction with a constant $\lambda \in[0,1)$. Let $x, y \in D$. Since $E(G)=D \times D,(x, y) \in E$ $(G)$. For each $x^{\prime} \in S(x)$, there exists $y^{\prime} \in S(y)$ such that $\left(x^{\prime}\right.$, $\left.y^{\prime}\right) \in E(G)$ and $d\left(x^{\prime}, y^{\prime}\right) \leq \lambda d(x, y)$. This implies that

$$
\sup _{x^{\prime} \in T(x)} \operatorname{dist}\left(x^{\prime}, S(y)\right) \leq \lambda d(x, y)
$$

Hence, $H(S(x), S(y)) \leq \lambda d(x, y)$.

The proof of (ii) is similar to (i).

A point $x \in D$ is said to be a fixed point of $S$ if and only if $x \in S(x)$. We shall denote by $F(S)$ the set of all fixed points of $S$. We say that $S$ satisfies the endpoint condition [11] if $F(S) \neq \varnothing$ and $S(x)=\{x\}$ for all $x \in F(S)$. In [9], the authors obtain a fixed-point theorem for a monotone increasing $G$-contraction mapping as the following result.

Theorem 2. Let $D$ be a nonempty closed subset of a complete metric space $(M, d)$ and $G=(V(G), E(G))$ a directed graph. Suppose that the following property holds:

(*) for any sequence $\left\{x_{n}\right\}$ in $D$, if $x_{n} \longrightarrow x$ and $\left(x_{n}, x_{n+1}\right)$ $\in E(G)$ for all $n \in \mathbb{N}$, then there exists a subsequence $\left\{x_{n_{k}}\right\}$ of $\left\{x_{n}\right\}$ such that $\left(x_{n_{k}}, x\right) \in E(G)$ for all $k \in \mathbb{N}$.

Let $S: D \longrightarrow C B(D)$ be a monotone increasing G-contraction mapping and $D_{S}:=\{x \in D:(x, y) \in E(G)$ for some $y$ $\in S(x)\}$. Then, $F(S) \neq \varnothing$ if and only if $D_{S} \neq \varnothing$.

Let $(M, d)$ be a metric space and $x, y \in M$. If $\phi$ is a mapping from a closed interval $[0, l]$ to $M$ such that $\phi(0)=$ $x, \phi(l)=y$, and $d(\phi(s), \phi(t))=|s-t|$ for all $s, t \in[0, l]$, then $\phi$ is called a geodesic joining $x$ to $y$. Furthermore, the image of $\phi$ is called a geodesic segment joining $x$ and $y$ which is denoted by $[x, y]$ whenever it is unique. If every two points of $M$ are joined by a geodesic, then $M$ is called a geodesic space. Moreover, if every two points of $M$ are joined by exactly one geodesic, then $M$ is called a uniquely geodesic space. For a subset $D$ of $M$, if every two points in $D$ can be joined by a geodesic in $M$ and the image of every such geodesic is contained in $D$, then we call $D$ a convex subset of $M$. A geodesic space $M$ is said to be a Hadamard space if $M$ is a complete metric space and for each $x, y, z \in M$, we have

$$
d^{2}(x, m) \leq \frac{1}{2} d^{2}(x, y)+\frac{1}{2} d^{2}(x, z)-\frac{1}{4} d^{2}(y, z)
$$

where $m$ is the midpoint of $[y, z]$. We refer the reader to [1214] for elementary properties of Hadamard spaces. It is known that every Hadamard space is uniquely geodesic. Notice also that $\mathbb{R}$-trees and Hilbert spaces are important examples of Hadamard spaces (see $[12,15]$ ). 
Let $D$ be a nonempty closed convex subset of a Hadamard space $(M, d)$. By Proposition 2.4 in [12], for each $x$ in $M$, there exists a unique point $x_{0}$ in $D$ such that

$$
d\left(x, x_{0}\right)=\inf \{d(x, y): y \in D\} .
$$

The metric projection of $M$ onto $D$ is the mapping $P_{D}: M \longrightarrow D$ defined by $P_{D}(x):=x_{0}$, where $x_{0}$ is the unique point in $D$ satisfying (7). From [16], for each $x, y$ $\in M$ and $t \in[0,1]$, there exists a unique point $z$ in $[x, y]$ such that

$$
d(x, z)=t d(x, y) \text { and } d(y, z)=(1-t) d(x, y) .
$$

The unique point $z$ satisfying (8) is denoted by ( $1-$ t) $x \oplus t y$.

Let $D$ be a nonempty convex subset of $M$ and $G=$ $(V(G), E(G))$ be a directed graph. We say that $G$ is convex if for any $x, y, z, w \in D$ and $t \in[0,1]$ such that $(x, z)$ and $(y, w)$ are in $E(G)$, we have $((1-t) x \oplus t y,(1-t) z \oplus t w) \in E(G)$.

Lemma 3 (see [16]). Let $(M, d)$ be a Hadamard space, $x, y$, $z \in M$ and $t \in[0,1]$. Then,

(i) $d((1-t) x \oplus t y, z) \leq(1-t) d(x, z)+t d(y, z)$

(ii) $d((1-t) x \oplus t y,(1-t) x \oplus t z) \leq t d(y, z)$

(iii) $d^{2}((1-t) x \oplus t y, z) \leq(1-t) d^{2}(x, z)+t d^{2}(y, z)-t$ $(1-t) d^{2}(x, y)$

For a bounded sequence $\left\{x_{n}\right\}$ in $M$, we define

$$
\begin{aligned}
r\left(\left\{x_{n}\right\}\right):=\inf \left\{\limsup _{n \rightarrow \infty} d\left(x_{n}, x\right): x \in M\right\}, \\
\mathscr{Z}\left(\left\{x_{n}\right\}\right):=\left\{x \in M: \limsup _{n \rightarrow \infty} d\left(x_{n}, x\right)=r\left(\left\{x_{n}\right\}\right)\right\} .
\end{aligned}
$$

The real number $r\left(\left\{x_{n}\right\}\right)$ and the set $\mathscr{Z}\left(\left\{x_{n}\right\}\right)$ are called the asymptotic radius and the asymptotic center of $\left\{x_{n}\right\}$, respectively. It is known that $\mathscr{Z}\left(\left\{x_{n}\right\}\right)$ consists of exactly one point (see, e.g., [17]). Let $\left\{x_{n}\right\}$ be a sequence in $M$ and $x \in M$. Then, $\left\{x_{n}\right\}$ is $\Delta$-convergent to $x$ if $\mathscr{E}\left(\left\{x_{n_{k}}\right\}\right)=\{x\}$ for every subsequence $\left\{x_{n_{k}}\right\}$ of $\left\{x_{n}\right\}$. In this case, we write $\Delta-\lim _{n \rightarrow \infty} x_{n}=x$.

Lemma 4. Let $(M, d)$ be a Hadamard space and $D$ a nonempty closed convex subset of $M$. Then,

(i) every bounded sequence in $M$ always has a $\Delta$-convergent subsequence [18]

(ii) the asymptotic center of any bounded sequence in $D$ belongs to D [19]
Let $(M, d)$ be a metric space. We use the notation $\overrightarrow{a b}$ for an ordered pair $(a, b)$ in $M \times M$. The quasilinearization is a mapping $\langle\cdot, \cdot\rangle:(M \times M) \times(M \times M) \longrightarrow \mathbb{R}$ which is defined by

$$
\begin{aligned}
\langle\overrightarrow{a b}, \overrightarrow{c e}\rangle:= & \frac{1}{2}\left[d^{2}(a, e)+d^{2}(b, c)-d^{2}(a, c)\right. \\
& \left.-d^{2}(b, e)\right] \text { for all } a, b, c, e \in M
\end{aligned}
$$

We say that $(M, d)$ satisfies the Cauchy-Schwarz inequality if

$$
|\langle\overrightarrow{a b}, \overrightarrow{c e}\rangle| \leq d(a, b) d(c, e) \text { for all } a, b, c, e \in M
$$

It is known that every Hadamard space satisfies the Cauchy-Schwarz inequality (see [20]).

Lemma 5. Let $(M, d)$ be a Hadamard space. Then, the following statements hold:

(i) Let $D$ be a nonempty closed convex subset of $M, x \in M$ and $z \in D$ [21]. Then,

$$
z=P_{D}(x) \quad \text { if and only if }\langle\overrightarrow{z x}, \overrightarrow{w z}\rangle \geq 0 \text { for all } w \in D
$$

(ii) Let $u, v \in M$. For each $t \in[0,1]$, we set $x_{t}=t u \oplus(1-$ t) $v$ [22]. Then, for $z \in M$, we have

$$
\left\langle\overrightarrow{x_{t} z}, \overrightarrow{x_{t} z}\right\rangle \leq t\left\langle\overrightarrow{u z}, \overrightarrow{x_{t} z}\right\rangle+(1-t)\left\langle\overrightarrow{v z}, \overrightarrow{x_{t} z}\right\rangle
$$

(iii) Let $\left\{x_{n}\right\}$ be a sequence in $M$ and $z \in M$ [23]. Then, $\left\{x_{n}\right\}$ is $\Delta$-convergent to $z$ if and only if $\limsup _{n \rightarrow \infty}\left\langle\overrightarrow{w z}, \overrightarrow{x_{n} z}\right\rangle \leq 0$ for all $w \in M$

We denote by $\ell_{\infty}$ the Banach space of bounded real sequences. Let $\mu$ be a continuous linear functional on $\ell_{\infty}$ and let $a=\left\{a_{1}, a_{2}, \cdots\right\} \in \ell_{\infty}$. We shall denote by $\mu_{n}\left(a_{n}\right)$ the value $\mu(a)$. A continuous linear functional $\mu$ on $\ell_{\infty}$ is called a Banach limit if $\|\mu\|=\mu(1,1, \cdots)=1$ and $\mu_{n}\left(a_{n}\right)=\mu_{n}\left(a_{n+1}\right)$ for all $\left\{a_{n}\right\} \in \ell_{\infty}$.

Lemma 6 (see [24]). Let $\left(a_{1}, a_{2}, \cdots\right) \in \ell^{\infty}$ be such that $\mu_{n}$ $\left(a_{n}\right) \leq 0$ for each Banach limit $\mu$ and suppose that lim $\sup _{n \rightarrow \infty}\left(a_{n+1}-a_{n}\right) \leq 0$. Then, $\lim \sup _{n \rightarrow \infty} a_{n} \leq 0$.

Lemma 7 (see [25]). Let $\left\{c_{n}\right\}$ be a sequence of nonnegative real numbers satisfying

$$
c_{n+1} \leq\left(1-\gamma_{n}\right) c_{n}+\gamma_{n} \eta_{n} \text { for all } n \in \mathbb{N}
$$

where $\left\{\gamma_{n}\right\} \subset(0,1)$ and $\left\{\eta_{n}\right\} \subset \mathbb{R}$ such that 
(i) $\sum_{n=1}^{\infty} \gamma_{n}=\infty$

(ii) $\sum_{n=1}^{\infty}\left|\gamma_{n} \eta_{n}\right|<\infty$ or limsup $\lim _{n \rightarrow \infty} \eta_{n} \leq 0$

Then, $\lim _{n \rightarrow \infty} c_{n}=0$.

\section{Main Results}

This section begins by proving a crucial lemma which is an extension of Lemma 2.1 in [26].

Lemma 8. Let $(M, d)$ be a Hadamard space, $D$ a nonempty closed convex subset of $M$, and $G=(V(G), E(G))$ a convex directed graph. Let $S: D \longrightarrow C B(D)$ be a monotone increasing $G$-nonexpansive mapping such that $u \in D_{S}$ for some $u \in D$. Suppose that $(*)$ holds. Then, for each $t \in(0,1)$, the mapping $S_{t}: D \longrightarrow C B(D)$ defined by

$$
S_{t}(x):=t u \oplus(1-t) S(x)
$$

has a fixed point $x_{t}$ in $D$; that is,

$$
x_{t} \in S_{t}\left(x_{t}\right)=t u \oplus(1-t) S\left(x_{t}\right) \text {. }
$$

Proof. Let $x, y \in D$ be such that $(x, y) \in E(G)$. Since $S$ is monotone increasing $G$-nonexpansive, for each $x^{\prime} \in S(x)$, there exists $y^{\prime} \in S(y)$ such that

$$
\begin{gathered}
\left(x^{\prime}, y^{\prime}\right) \in E(G), \\
d\left(x^{\prime}, y^{\prime}\right) \leq d(x, y) .
\end{gathered}
$$

By the convexity of $G$, we have

$$
\left(t u \oplus(1-t) x^{\prime}, t u \oplus(1-t) y^{\prime}\right) \in E(G)
$$

By (17) and Lemma 3 (ii), we have

$$
\begin{aligned}
d\left(t u \oplus(1-t) x^{\prime}, t u \oplus(1-t) y^{\prime}\right) & \leq(1-t) d\left(x^{\prime}, y^{\prime}\right) \\
& \leq(1-t) d(x, y)
\end{aligned}
$$

This implies that $S_{t}$ is monotone increasing G-contraction. Since $u \in D_{S}$, there exists $v \in S(u)$ such that $(u, v) \in E$ $(G)$. By the convexity of $G$, we have $(u, t u \oplus(1-t) v) \in E(G)$. This implies that $u \in D_{S_{t}}$ and hence $D_{S_{t}} \neq \varnothing$. By Theorem 2, $S_{t}$ has a fixed point $x_{t}$ in $D$.

In [6], the authors introduce a property that is stronger than the condition $(*)$ in Lemma 8.

Definition 9. Let $(M, d)$ be a Hadamard space, $D$ a nonempty subset of $M$, and $G=(V(G), E(G))$ a directed graph. Then, $D$ is said to have property $G$ if for any sequence $\left\{x_{n}\right\}$ in $D$ such that $\Delta-\lim _{n \rightarrow \infty} x_{n}=x \in D$, there exists a subsequence $\left\{x_{n_{k}}\right\}$ of $\left\{x_{n}\right\}$ such that $\left(x_{n_{k}}, x\right) \in E(G)$ for all $k \in \mathbb{N}$.
The following result is an extension of Lemma 3.2 in [27].

Lemma 10. Let $(M, d)$ be a Hadamard space and $D$ a nonempty closed convex subset of $M$. Let $G=(V(G), E(G))$ be a directed graph and $S: D \rightarrow K(M)$ a monotone increasing $G$-nonexpansive mapping and $\left\{x_{n}\right\}$ a bounded sequence in D. Suppose that $D$ has property $G$. If $\lim _{n \rightarrow \infty} \operatorname{dist}\left(x_{n}\right.$, $\left.S\left(x_{n}\right)\right)=0$ and $\Delta-\lim _{n \rightarrow \infty} x_{n}=x$, then $x \in S(x)$.

Proof. For $n \in \mathbb{N}$, let $y_{n} \in S\left(x_{n}\right)$ be such that $d\left(x_{n}, y_{n}\right)=$ dist $\left(x_{n}, S\left(x_{n}\right)\right)$. By Lemma 4 (ii), $x \in D$. Since $D$ has property $G$, there exists a subsequence $\left\{x_{n_{k}}\right\}$ of $\left\{x_{n}\right\}$ such that $\left(x_{n_{k}}, x\right) \in E(G)$ for all $k \in \mathbb{N}$. Since $S$ is monotone increasing $G$-nonexpansive, for each $k \in \mathbb{N}$ there exists $z_{n_{k}} \in S(x)$ such that

$$
\begin{gathered}
\left(y_{n_{k}}, z_{n_{k}}\right) \in E(G), \\
d\left(y_{n_{k}}, z_{n_{k}}\right) \leq d\left(x_{n_{k}}, x\right) .
\end{gathered}
$$

Since $S(x)$ is compact, without loss of generality, we may assume that $z_{n_{k}} \longrightarrow z \in S(x)$. Thus,

$$
\begin{aligned}
d\left(x_{n_{k}}, z\right) & \leq d\left(x_{n_{k}}, y_{n_{k}}\right)+d\left(y_{n_{k}}, z_{n_{k}}\right)+d\left(z_{n_{k}}, z\right) \\
& \leq \operatorname{dist}\left(x_{n_{k}}, S\left(x_{n_{k}}\right)\right)+d\left(x_{n_{k}}, x\right)+d\left(z_{n_{k}}, z\right) .
\end{aligned}
$$

This implies that $\limsup _{k \rightarrow \infty} d\left(x_{n_{k}}, z\right) \leq \limsup _{k \rightarrow \infty} d\left(x_{n_{k}}\right.$, $x)$. Thus, $z \in \mathscr{Z}\left(\left\{x_{n_{k}}\right\}\right)=\{x\}$ and hence, $x=z \in S(x)$.

By applying Lemmas 8 and 10, we can obtain a fixedpoint theorem for monotone increasing $G$-nonexpansive mappings in Hadamard spaces.

Theorem 11. Let $(M, d)$ be a Hadamard space and $D$ a nonempty bounded closed convex subset of X. Let $G=(V(G)$, $E(G))$ be a convex directed graph and suppose that $D$ has property $G$. Let $S: D \longrightarrow K(D)$ be a monotone increasing $G$-nonexpansive mapping such that $u \in D_{S}$ for some $u \in D$. Then, $S$ has a fixed point in $D$.

Proof. For each $n \in \mathbb{N}$, we define a mapping $S_{n}: D \longrightarrow K(D)$ by

$$
S_{n}(x):=\frac{1}{n} u \oplus\left(1-\frac{1}{n}\right) S(x) \text { for all } x \in D \text {. }
$$

Then, by Lemma 8 , there exists $x_{n}$ in $D$ such that $x_{n} \in$ $S_{n}\left(x_{n}\right)=(1 / n) u \oplus(1-1 / n) S\left(x_{n}\right)$. For each $n \in \mathbb{N}$, there exists $y_{n} \in S\left(x_{n}\right)$ such that

$$
x_{n}=\frac{1}{n} u \oplus\left(1-\frac{1}{n}\right) y_{n} .
$$


This implies that

$$
\operatorname{dist}\left(x_{n}, S\left(x_{n}\right)\right) \leq d\left(x_{n}, y_{n}\right)=\frac{1}{n} d\left(u, y_{n}\right) \longrightarrow 0 \text { as } n \longrightarrow \infty .
$$

Since $\left\{x_{n}\right\}$ is bounded, by Lemma 4 , there exists a subsequence $\left\{x_{n_{k}}\right\}$ of $\left\{x_{n}\right\}$ and a point $x$ in $D$ such that $\Delta-$ $\lim _{k \rightarrow \infty} x_{n_{k}}=x$. By Lemma 10, we have $x \in S(x)$ and hence, the proof is complete.

As a consequence of Theorem 11, by putting $V(G)=D$ and $E(G)=D \times D$, we obtain a result of Dhompongsa et al. [28].

Corollary 12. Let $(M, d)$ be a Hadamard space and $D$ a nonempty bounded closed convex subset of $M$. Let $S: D \longrightarrow K(D)$ be a nonexpansive mapping. Then, $S$ has a fixed point.

The following result gives conditions that guarantee the closedness and the convexity of the fixed-point set of a monotone increasing $G$-nonexpansive mapping on a closed convex subset of a Hadamard space. Notice also that it is an extension of Theorem 3.2 in [6].

Lemma 13. Let $(M, d)$ be a Hadamard space and $D$ a nonempty closed convex subset of $M$. Let $G=(V(G), E(G))$ be a convex directed graph and suppose that $D$ has property $G$. If $S: D \longrightarrow C B(D)$ is a monotone increasing $G$-nonexpansive mapping satisfying the endpoint condition and $F(S) \times F(S)$ $\subseteq E(G)$, then $F(S)$ is closed and convex.

Proof. Let $\left\{x_{n}\right\}$ be a sequence in $F(S)$ such that $\lim _{n \rightarrow \infty} x_{n}=x$. Since $D$ has property $G$, there is a subsequence $\left\{x_{n_{k}}\right\}$ of $\left\{x_{n}\right\}$ such that $\left(x_{n_{k}}, x\right) \in E(G)$ for all $k \in \mathbb{N}$. Since $S$ is monotone increasing $G$-nonexpansive, there exists $z_{n_{k}} \in S(x)$ such that

$$
\begin{gathered}
\left(x_{n_{k}}, z_{n_{k}}\right) \in E(G), \\
d\left(x_{n_{k}}, z_{n_{k}}\right) \leq d\left(x_{n_{k}}, x\right) .
\end{gathered}
$$

Thus

$$
\begin{aligned}
\operatorname{dist}(x, S(x)) & \leq d\left(x, z_{n_{k}}\right) \leq d\left(x, x_{n_{k}}\right)+d\left(x_{n_{k}}, z_{n_{k}}\right) \\
& \leq 2 d\left(x_{n_{k}}, x\right) \longrightarrow 0 \text { as } k \longrightarrow \infty .
\end{aligned}
$$

This implies that $x \in S(x)$ and hence, $F(S)$ is closed. Let $x, y \in F(S)$ and $z=\alpha x \oplus(1-\alpha) y$ for some $\alpha \in(0,1)$. Since $F$ $(S) \times F(S) \subseteq E(G)$, both $(x, x)$ and $(y, x)$ belong to $E(G)$. By the convexity of $G$, we have $(z, x) \in E(G)$. Take any point $v$ $\in S(z)$. Since $S$ is monotone increasing $G$-nonexpansive, there exists $w \in S(x)=\{x\}$ such that $(v, w) \in E(G)$ and $d(v, w) \leq d$ $(z, x)$. This implies that $d(v, x) \leq d(z, x)$. Similarly, we can show that $d(v, y) \leq d(z, y)$. It follows from Lemma 3 (iii) that

$$
\begin{aligned}
d^{2}(v, z) \leq & \alpha d^{2}(v, x)+(1-\alpha) d^{2}(v, y)-\alpha(1-\alpha) d^{2}(x, y) \\
\leq & \alpha d^{2}(z, x)+(1-\alpha) d^{2}(z, y)-\alpha(1-\alpha) d^{2}(x, y) \\
\leq & \alpha(1-\alpha)^{2} d^{2}(x, y)+(1-\alpha) \alpha^{2} d^{2}(x, y) \\
& -\alpha(1-\alpha) d^{2}(x, y)=0,
\end{aligned}
$$

which yields $z=v \in S(z)$. Therefore, $z \in F(S)$ and hence, $F(S)$ is convex.

Now, we prove the strong convergence of Browder's iteration for monotone increasing $G$-nonexpansive mappings in Hadamard spaces.

Theorem 14. Let $(M, d)$ be a Hadamard space and $D$ a nonempty closed convex subset of $M$. Let $G=(V(G), E(G))$ be a convex directed graph and suppose that $D$ has property $G$. Let $S: D \longrightarrow K(D)$ be a monotone increasing G-nonexpansive mapping satisfying the endpoint condition and having $u \in D_{S}$ for some $u \in D$. Suppose that $F(S) \times F(S)$ $\subseteq E(G)$. Then, the following statements hold:

(i) If the net $\left\{x_{t}\right\}$ defined by (16) dominates $F(S)$, then $\left\{x_{t}\right\}$ converges strongly to $P_{F(S)}(u)$ as $t \longrightarrow 0$

(ii) If $\left\{u_{n}\right\}$ is a bounded sequence in $D$ such that $\lim _{n \rightarrow \infty} \operatorname{dist}\left(u_{n}, S\left(u_{n}\right)\right)=0$, then $d^{2}\left(u, P_{F(S)}(u)\right) \leq \mu_{n}$ $d^{2}\left(u, u_{n}\right)$ for each Banach limit $\mu$

Proof. (i) We first show that $\left\{x_{t}\right\}$ is bounded. Let $p \in F(S)$. Since $\left\{x_{t}\right\}$ dominates $F(S),\left(x_{t}, p\right) \in E(G)$. From (16), there exists $y_{t} \in S\left(x_{t}\right)$ such that $x_{t}=t u \oplus(1-t) y_{t}$. It follows from the $G$-nonexpansiveness of $S$ and the endpoint condition that $\left(y_{t}, p\right) \in E(G)$ and $d\left(y_{t}, p\right) \leq d\left(x_{t}, p\right)$. This yields

$d\left(x_{t}, p\right) \leq t d(u, p)+(1-t) d\left(y_{t}, p\right) \leq t d(u, p)+(1-t) d\left(x_{t}, p\right)$,

which implies that $d\left(x_{t}, p\right) \leq d(u, p)$ and hence, $\left\{x_{t}\right\}$ is bounded. Notice also that $\left\{y_{t}\right\}$ is bounded and $\operatorname{dist}\left(x_{t}\right.$, $\left.S\left(x_{t}\right)\right) \leq d\left(x_{t}, y_{t}\right) \leq t d\left(u, y_{t}\right) \longrightarrow 0$ as $t \longrightarrow 0$.

Next, we show that $\left\{x_{t}\right\}$ converges strongly to $P_{F(S)}(u)$ as $t \longrightarrow 0$. Let a sequence $\left\{t_{n}\right\}$ in $(0,1)$ converging to 0 and put $x_{n}:=x_{t_{n}}$. It suffices to show that there exists a subsequence of $\left\{x_{n}\right\}$ which converges to $P_{F(S)}(u)$. By Lemmas 4 and 10, there exists a subsequence $\left\{x_{n_{k}}\right\}$ of $\left\{x_{n}\right\}$ and a point $z$ in $F(S)$ such that $\Delta-\lim _{k \rightarrow \infty} x_{n_{k}}=z$. By Lemma 5 (ii), we have

$$
\begin{aligned}
d^{2}\left(x_{n_{k}}, z\right)= & \left\langle\overrightarrow{x_{n_{k}} z}, \overrightarrow{x_{n_{k}} z}\right\rangle \leq t_{n_{k}}\left\langle\overrightarrow{u z}, \overrightarrow{x_{n_{k}} z}\right\rangle \\
& +\left(1-t_{n_{k}}\right)\left\langle\overrightarrow{y_{n_{k}} z}, \overrightarrow{x_{n_{k}} z}\right\rangle \leq t_{n_{k}}\left\langle\overrightarrow{u z}, \overrightarrow{x_{n_{k}} z}\right\rangle \\
& +\left(1-t_{n_{k}}\right) d\left(y_{n_{k}}, z\right) d\left(x_{n_{k}}, z\right) \leq t_{n_{k}}\left\langle\overrightarrow{u z}, \overrightarrow{x_{n_{k}} z}\right\rangle \\
& +\left(1-t_{n_{k}}\right) d^{2}\left(x_{n_{k}}, z\right) .
\end{aligned}
$$


Thus, $d^{2}\left(x_{n_{k}}, z\right) \leq\left\langle\overrightarrow{u z}, \overrightarrow{x_{n_{k}}} \vec{z}\right\rangle$. Since $\Delta-\lim _{k \rightarrow \infty} x_{n_{k}}=z$, by Lemma 5 (iii), we have $\limsup _{k \rightarrow \infty}\left\langle\overrightarrow{u z}, \overrightarrow{x_{n_{k}}} \vec{z}\right\rangle \leq 0$ and this in turn implies that $\lim _{k \rightarrow \infty} x_{n_{k}}=z$. Next, we show that $z=P_{F(S)}$ $(u)$. Since $\left\{x_{n_{k}}\right\}$ dominates $F(S)$, for any $q \in F(S)$, we have

$$
\begin{aligned}
d^{2}\left(x_{n_{k}}, q\right) \leq & t_{n_{k}} d^{2}(u, q)+\left(1-t_{n_{k}}\right) d^{2}\left(y_{n_{k}}, q\right) \\
& -t_{n_{k}}\left(1-t_{n_{k}}\right) d^{2}\left(u, y_{n_{k}}\right) \leq t_{n_{k}} d^{2}(u, q) \\
& +\left(1-t_{n_{k}}\right) d^{2}\left(x_{n_{k}}, q\right)-t_{n_{k}}\left(1-t_{n_{k}}\right) d^{2}\left(u, y_{n_{k}}\right)
\end{aligned}
$$

Thus,

$$
d^{2}\left(x_{n_{k}}, q\right) \leq d^{2}(u, q)-\left(1-t_{n_{k}}\right) d^{2}\left(u, y_{n_{k}}\right) .
$$

Let $k \longrightarrow \infty$, then

$$
d^{2}(z, q) \leq d^{2}(u, q)-d^{2}(u, z) .
$$

Hence,

$$
\begin{aligned}
0 & \leq \frac{1}{2}\left[d^{2}(z, z)+d^{2}(u, q)-d^{2}(z, q)-d^{2}(u, z)\right] \\
& =\langle\overrightarrow{z u}, \overrightarrow{q z}\rangle \text { for all } q \in F(S) .
\end{aligned}
$$

By Lemma 5 (i), $z=P_{F(S)}(u)$. In summary, for any given sequence $\left\{t_{n}\right\}$ in $(0,1)$ converging to 0 , the sequence $\left\{x_{n}:=\right.$ $\left.x_{t_{n}}\right\}$ has a subsequence converging to $P_{F(S)}(u)$. This shows that $x_{n} \longrightarrow P_{F(S)}(u)$ and in turn implies that $x_{t} \longrightarrow P_{F(S)}(u)$ as $t \longrightarrow 0$.

(ii) The proof is similar to the proof of Theorem 3.4 in [27], so we omit it.

Now, we give an example which supports Theorem 14 .

Example 1. Let $M=(\mathbb{R},|\cdot|), D=[0,1]$ and let $G=(V(G), E$ $(G)$ ) be such that $V(G)=[0,1 / 2]$ and $E(G)=\{(x, y): x, y \in$ $[0,1 / 2]\}$. It is easy to see that $G$ is convex. Let $S: D \longrightarrow K(D)$ be defined by

$$
S(x):=\left(\begin{array}{ll}
\left\{\frac{x}{2}\right\} & \text { if } x \neq 1, \\
\{0\} & \text { if } x=1 .
\end{array}\right.
$$

Then, $S$ is a monotone increasing $G$-nonexpansive mapping satisfying the endpoint condition. Notice also that $F(S)=\{0\}$. Thus, $F(S) \times F(S) \subseteq E(G)$. Fix $u \in D_{S}=[0,1 / 2]$. For each $t \in(0,1)$, let $x_{t}$ be defined by (16). Obviously, $\left\{x_{t}\right\}$ dominates $F(S)$ and

$$
x_{t}=t u+(1-t) \frac{x_{t}}{2} .
$$

Thus, $x_{t} / 2=t\left(u-x_{t} / 2\right) \longrightarrow 0$ as $t \longrightarrow 0$. This implies that $x_{t} \longrightarrow 0=P_{F(S)}(u)$. However, $S$ is not nonexpansive since

$$
H\left(S(1), S\left(\frac{3}{4}\right)\right)=\left|0-\frac{3}{8}\right|=\frac{3}{8}>\frac{1}{4}=\left|1-\frac{3}{4}\right| .
$$

As a consequence of Theorem 14, by putting $V(G)=D$ and $E(G)=D \times D$, we obtain a result of Dhompongsa et al. [27].

Corollary 15. Let $(M, d)$ be a Hadamard space, $D$ a nonempty closed convex subset of $M$, and $S: D \rightarrow K(D)$ a nonexpansive mapping satisfying the endpoint condition. Fix $u \in D$; for each $t \in(0,1)$, let $x_{t}$ be a fixed point of $S_{t}: D \longrightarrow K(D)$ defined by

$$
S_{t}(x):=t u \oplus(1-t) S(x), \text { for all } x \in D .
$$

Then, the following statements hold:

(i) $\left\{x_{t}\right\}$ converges strongly to $P_{F(S)}(u)$ as $t \longrightarrow 0$

(ii) If $\left\{u_{n}\right\}$ is a bounded sequence in $D$ such that $\lim _{n \rightarrow \infty} \operatorname{dist}\left(u_{n}, S\left(u_{n}\right)\right)=0$, then $d^{2}\left(u, P_{F(S)}(u)\right) \leq \mu_{n}$ $d^{2}\left(u, u_{n}\right)$ for each Banach limit $\mu$

Next, we will extend the strong convergence of Halpern's iteration from single-valued $G$-nonexpansive mappings [7] to the general setting of multivalued $G$-nonexpansive mappings. Notice that our approach does not require the transitivity of the graph $G$.

Let $(M, d)$ be a Hadamard space and $D$ a nonempty closed convex subset of $M$. Let $G=(V(G), E(G))$ be a convex directed graph and $S: D \longrightarrow K(D)$ a monotone increasing $G$-nonexpansive mapping for which $D_{S} \neq \varnothing$. Let $\left\{\alpha_{n}\right\}$ be a sequence in $(0,1)$. Fix $u \in D_{S}$, and let $x_{1}=u$. Then, there exists $y_{1} \in S\left(x_{1}\right)$ such that $\left(x_{1}, y_{1}\right) \in E(G)$. Let

$$
x_{2}=\alpha_{1} u \oplus\left(1-\alpha_{1}\right) y_{1} .
$$

Since $\left(x_{1}, x_{1}\right) \in E(G)$ and $G$ is convex, $\left(x_{1}, x_{2}\right) \in E(G)$. Since $S$ is monotone increasing $G$-nonexpansive, there exists $y_{2} \in S\left(x_{2}\right)$ such that $\left(y_{1}, y_{2}\right) \in E(G)$ and $d\left(y_{1}, y_{2}\right) \leq$ $d\left(x_{1}, x_{2}\right)$. Let

$$
x_{3}=\alpha_{2} u \oplus\left(1-\alpha_{2}\right) y_{2} .
$$

Since $(u, u) \in E(G)$ and $\left(y_{1}, y_{2}\right) \in E(G)$, by the convexity of $G$, we have $\left(x_{2}, x_{3}\right) \in E(G)$. Inductively, we can construct sequences $\left\{x_{n}\right\}$ and $\left\{y_{n}\right\}$ in $D$ such that

$$
\begin{aligned}
x_{n+1} & =\alpha_{n} u \oplus\left(1-\alpha_{n}\right) y_{n}, y_{n} \in S\left(x_{n}\right), \\
\left(y_{n}, y_{n+1}\right) & \in E(G), \\
\left(x_{n}, x_{n+1}\right) & \in E(G), \\
d\left(y_{n}, y_{n+1}\right) & \leq d\left(x_{n}, x_{n+1}\right) \text { for all } n \in \mathbb{N} .
\end{aligned}
$$

Theorem 16. Let $(M, d)$ be a Hadamard space and $D$ a nonempty closed convex subset of $M$. Let $G=(V(G)$, $E(G))$ be a convex directed graph and suppose that $D$ has property $G$. Let $S: D \longrightarrow K(D)$ be a monotone increasing 
G-nonexpansive mapping satisfying the endpoint condition and having $u \in D_{S}$ for some $u \in D$. Suppose that $F(S) \times$ $F(S) \subseteq E(G)$, and let $\left\{\alpha_{n}\right\}$ be a sequence in $(0,1)$ satisfying

(C1) $\lim _{n \rightarrow \infty} \alpha_{n}=0$

(C2) $\sum_{n=1}^{\infty} \alpha_{n}=\infty$,

(C3) $\sum_{n=1}^{\infty}\left|\alpha_{n}-\alpha_{n+1}\right|<\infty$ or $\lim _{n \rightarrow \infty}\left(\alpha_{n} / \alpha_{n+1}\right)=1$

For each $n \in \mathbb{N}$, let $x_{n}$ be defined by (40) and suppose that $\left\{x_{n}\right\}$ dominates $P_{F(S)}(u)$. Then, $\left\{x_{n}\right\}$ converges strongly to $P_{F(S)}(u)$.

Proof. We divide the proof into three steps.

Step 1. We show that $\left\{x_{n}\right\}$ and $\left\{y_{n}\right\}$ are bounded sequences. Let $z:=P_{F(S)}(u)$. Since $\left\{x_{n}\right\}$ dominates $z,\left(x_{n}, z\right) \in E(G)$. It follows from the $G$-nonexpansiveness of $S$ and the endpoint condition that $\left(y_{n}, z\right) \in E(G)$ and $d\left(y_{n}, z\right) \leq d\left(x_{n}, z\right)$. Thus,

$$
\begin{aligned}
d\left(x_{n+1}, z\right) \leq & \alpha_{n} d(u, z)+\left(1-\alpha_{n}\right) d\left(y_{n}, z\right) \leq \alpha_{n} d(u, z) \\
& +\left(1-\alpha_{n}\right) d\left(x_{n}, z\right) \leq \max \left\{d(u, z), d\left(x_{n}, z\right)\right\}
\end{aligned}
$$

for all $n \in \mathbb{N}$. This implies that $\left\{x_{n}\right\}$ is bounded and so is $\left\{y_{n}\right\}$.

Step 2. We show that $\lim _{n \rightarrow \infty} \operatorname{dist}\left(x_{n}, S\left(x_{n}\right)\right)=0$. It follows from (40)-(42) that

$$
\begin{aligned}
d\left(x_{n}, x_{n+1}\right)= & d\left(\alpha_{n-1} u \oplus\left(1-\alpha_{n-1}\right) y_{n-1}, \alpha_{n} u \oplus\left(1-\alpha_{n}\right) y_{n}\right) \\
\leq & d\left(\alpha_{n-1} u \oplus\left(1-\alpha_{n-1}\right) y_{n-1}, \alpha_{n} u \oplus\left(1-\alpha_{n}\right) y_{n-1}\right) \\
& +d\left(\alpha_{n} u \oplus\left(1-\alpha_{n}\right) y_{n-1}, \alpha_{n} u \oplus\left(1-\alpha_{n}\right) y_{n}\right) \\
\leq & \left|\alpha_{n-1}-\alpha_{n}\right| d\left(u, y_{n-1}\right)+\left(1-\alpha_{n}\right) d\left(y_{n-1}, y_{n}\right) \\
\leq & \left(1-\alpha_{n}\right) d\left(x_{n-1}, x_{n}\right)+\left|\alpha_{n-1}-\alpha_{n}\right| d\left(u, y_{n-1}\right) .
\end{aligned}
$$

Putting, in Lemma 7, $c_{n}=d\left(x_{n-1}, x_{n}\right), \gamma_{n}=\alpha_{n}$ and $\eta_{n}=1$ $1-\left(\alpha_{n-1} / \alpha_{n}\right) \mid d\left(u, y_{n-1}\right)$, we get by (C2) and (C3) that $\lim _{n \rightarrow \infty} d\left(x_{n}, x_{n+1}\right)=0$. Consequently, by $(\mathrm{C} 1)$, we have

$$
\begin{aligned}
\operatorname{dist}\left(x_{n}, S\left(x_{n}\right)\right) & \leq d\left(x_{n}, y_{n}\right) \leq d\left(x_{n}, x_{n+1}\right)+d\left(x_{n+1}, y_{n}\right) \\
& =d\left(x_{n}, x_{n+1}\right)+d\left(\alpha_{n} u \oplus\left(1-\alpha_{n}\right) y_{n}, y_{n}\right) \\
& \leq d\left(x_{n}, x_{n+1}\right)+\alpha_{n} d\left(u, y_{n}\right) \longrightarrow 0 \text { as } n \longrightarrow \infty
\end{aligned}
$$

Step 3. We show that $\left\{x_{n}\right\}$ converges strongly to $z$. By Lemma 3 (iii), we get that

$$
\begin{aligned}
d^{2}\left(x_{n+1}, z\right) \leq & \alpha_{n} d^{2}(u, z)+\left(1-\alpha_{n}\right) d^{2}\left(y_{n}, z\right) \\
& -\alpha_{n}\left(1-\alpha_{n}\right) d^{2}\left(u, y_{n}\right) \leq\left(1-\alpha_{n}\right) d^{2}\left(x_{n}, z\right) \\
& +\alpha_{n}\left(d^{2}(u, z)-\left(1-\alpha_{n}\right) d^{2}\left(u, y_{n}\right)\right) .
\end{aligned}
$$

By Theorem 14 (ii), we have $\mu_{n}\left(d^{2}(u, z)-d^{2}\left(u, x_{n}\right)\right) \leq 0$ for each Banach limit $\mu$. Moreover, since $\lim _{n \rightarrow \infty} d\left(x_{n}\right.$, $\left.x_{n+1}\right)=0$,

$$
\limsup _{n \rightarrow \infty}\left[\left(d^{2}(u, z)-d^{2}\left(u, x_{n+1}\right)\right)-\left(d^{2}(u, z)-d^{2}\left(u, x_{n}\right)\right)\right]=0 .
$$

It follows from Lemma 6 that

$$
\begin{aligned}
\underset{n \rightarrow \infty}{\limsup } & {\left[\left(d^{2}(u, z)-\left(1-\alpha_{n}\right) d^{2}\left(u, y_{n}\right)\right]\right.} \\
& =\limsup _{n \rightarrow \infty}\left[\left(d^{2}(u, z)-d^{2}\left(u, x_{n}\right)\right] \leq 0 .\right.
\end{aligned}
$$

Applying Lemma 7 to the inequality (46), we can conclude that $x_{n} \longrightarrow z$ as $n \longrightarrow \infty$, and hence, the proof is complete.

\section{Concluding Remarks and Open Questions}

In this paper, we show that if $D$ is a nonempty subset of a metric space and $G=(V(G), E(G))$ is a directed graph such that $V(G)=D$ and $E(G)=D \times D$, then the concept of monotone increasing $G$-nonexpansive mappings introduced by Alfuraidan and Khamsi [9] coincides with the concept of multivalued nonexpansive mappings. Moreover, by using the concept of property $G$ introduced by Tiammee et al. [6], we prove the analogue results of Theorem 3.4 in [27], Theorem 3.2 in [6], and Theorem 3.6 in [7] in the setting of monotone increasing $G$-nonexpansive mappings in Hadamard spaces endowed with graphs. However, in view of Example 1, we still have the strong convergence result of Browder's iteration for a monotone increasing $G$-nonexpansive mapping $T$ on a set $D$ which has no property $G$. Thus, it is unclear whether property $G$ is necessary for Theorem 14. Therefore, the following question should be of interest.

Question 1. Can we omit property $G$ from Theorem 14 ?

\section{Data Availability}

No data were used to support this study.

\section{Conflicts of Interest}

The author declares that there are no conflicts of interest.

\section{Acknowledgments}

The author thanks Dr. Attapol Kaewkhao for a useful discussion. This research was supported by Chiang Mai University.

\section{References}

[1] S. Banach, "Sur les opérations dans les ensembles abstraits et leur application aux équations intégrales," Fundamenta Mathematicae, vol. 3, pp. 133-181, 1922. 
[2] F. E. Browder, "Convergence of approximants to fixed points of nonexpansive nonlinear mappings in Banach spaces," Archive for Rational Mechanics and Analysis, vol. 24, no. 1, pp. 82-90, 1967.

[3] B. Halpern, "Fixed points of nonexpanding maps," Bulletin of the American Mathematical Society, vol. 73, no. 6, pp. 957962, 1967.

[4] R. P. Agarwal, D. O'Regan, and D. R. Sahu, Fixed Point Theory for Lipschitzian-Type Mappings with Applications, Topological Fixed Point Theory and Its Applications, 6, Springer, New York, NY, USA, 2009.

[5] J. Jachymski, "The contraction principle for mappings on a metric space with a graph," Proceedings of the American Mathematical Society, vol. 136, no. 4, pp. 1359-1373, 2008.

[6] J. Tiammee, A. Kaewkhao, and S. Suantai, “On Browder's convergence theorem and Halpern iteration process for $G$-nonexpansive mappings in Hilbert spaces endowed with graphs," Fixed Point Theory and Applications, vol. 2015, Article ID $187,2015$.

[7] W. Anakkamatee and B. Tongnoi, "Convergence theorems for $G$-nonexpansive mappings in CAT(0) spaces endowed with graphs," Thai Journal of Mathematics, vol. 17, pp. 775-787, 2019.

[8] I. Beg, A. R. Butt, and S. Radojevic, "The contraction principle for set valued mappings on a metric space with a graph," Computers \& Mathematcs with Applications, vol. 60, no. 5, pp. 1214-1219, 2010.

[9] M. R. Alfuraidan and M. A. Khamsi, "Fixed points of monotone nonexpansive mappings on a hyperbolic metric space with a graph," Fixed Point Theory and Applications, vol. 2015, no. 1, 2015.

[10] H. K. Xu, "On weakly nonexpansive and *-nonexpansive multivalued mappings," Math Japonica, vol. 36, pp. 441-445, 1991.

[11] N. Shahzad and J. Markin, "Invariant approximations for commuting mappings in CAT(0) and hyperconvex spaces," Journal of Mathematical Analysis and Applications, vol. 337, no. 2, pp. 1457-1464, 2008.

[12] M. Bridson and A. Haefliger, Metric Spaces of Non-Positive Curvature, Springer-Verlag, Berlin, Heidelberg, 1999.

[13] D. Burago, Y. Burago, and S. Ivanov, A Course in Metric Geometry, Graduate Studies in Math. vol. 33, American Mathematical Society, Providence, RI, USA, 2001.

[14] B. Panyanak and S. Suantai, "Diametrically regular mappings and Browder's theorem without the endpoint condition," Numerical Functional Analysis and Optimization, vol. 41, no. 4, pp. 495-505, 2020.

[15] K. S. Brown, Buildings, Springer-Verlag, New York, NY, USA, 1989.

[16] S. Dhompongsa and B. Panyanak, "On $\triangle$-convergence theorems in CAT(0) spaces," Computers \& Mathematcs with Applications, vol. 56, no. 10, pp. 2572-2579, 2008.

[17] S. Dhompongsa, W. A. Kirk, and B. Sims, "Fixed points of uniformly Lipschitzian mappings," Nonlinear Analysis, vol. 65, no. 4, pp. 762-772, 2006.

[18] W. A. Kirk and B. Panyanak, "A concept of convergence in geodesic spaces," Nonlinear Analysis, vol. 68, no. 12, pp. 3689-3696, 2008.

[19] S. Dhompongsa, W. A. Kirk, and B. Panyanak, "Nonexpansive set-valued mappings in metric and Banach spaces," Journal of Nonlinear and Convex Analysis, vol. 8, pp. 35-45, 2007.
[20] I. D. Berg and I. G. Nikolaev, "Quasilinearization and curvature of Aleksandrov spaces," Geometriae Dedicata, vol. 133, no. 1, pp. 195-218, 2008.

[21] H. Dehghan and J. Rooin, "Metric projection and convergence theorems for nonexpansive mappings in Hadamard spaces," Journal of Nonlinear and Convex Analysis, 2018.

[22] R. Wangkeeree and P. Preechasilp, "Viscosity approximation methods for nonexpansive mappings in CAT(0) spaces," Journal of Inequalities and Applications, vol. 2013, Article ID 93, 2013.

[23] B. A. Kakavandi, "Weak topologies in complete CAT(0) metric spaces," Proceedings of the American Mathematical Society, vol. 141, no. 3, pp. 1029-1039, 2013.

[24] N. Shioji and W. Takahashi, "Strong convergence of approximated sequences for nonexpansive mappings in Banach spaces," Proceedings of the American Mathematical Society, vol. 125, no. 12, pp. 3641-3646, 1997.

[25] H. K. Xu, "An iterative approach to quadratic optimization," Journal of Optimization Theory and Applications, vol. 116, no. 3, pp. 659-678, 2003.

[26] S. Saejung, "Halpern's iteration in CAT(0) spaces," Fixed Point Theory and Applications, vol. 2010, Article ID 471781, 13 pages, 2010.

[27] S. Dhompongsa, A. Kaewkhao, and B. Panyanak, "On Kirk's strong convergence theorem for multivalued nonexpansive mappings on CAT(0) spaces," Nonlinear Analysis, vol. 75, no. 2, pp. 459-468, 2012.

[28] S. Dhompongsa, A. Kaewkhao, and B. Panyanak, "Lim's theorems for multivalued mappings in CAT(0) spaces," Journal of Mathematical Analysis and Applications, vol. 312, no. 2, pp. 478-487, 2005. 